\title{
Combining Biocatalysts and Semi-Synthesis to (Bio) Synthesize Recalcitrant Pharmaceuticals
}

\author{
Nik Kovinich* \\ Department of Plant and Soil Sciences, USA
}

*Corresponding author: Nik Kovinich, Department of Plant and Soil Sciences, USA.

Received Date: May 10, 2019

Published Date: May 17, 2019

\section{Abstract}

Difficult-to-synthesize (recalcitrant) bioactive molecules can be (bio)synthesized using semi-synthesis and biocatalysis methods in tandem. Biocatalysts modules consist of promiscuous enzymes heterologously expressed in microbes that can accept semi-synthetic versions of natural products. The ordering of semi-synthesis and biocatalysis reactions can be changed to optimize the overall efficiency of (bio)synthesis.

\section{Short Communication}

Most small molecules that were approved as anticancer drugs by the U.S. Food and Drug Administration (FDA) between 1981 and 2014 were derivatives of natural products (NPs) [1]. The most common approach for manufacturers to produce these molecules is by semi- or full synthesis. Despite the high efficiency and amenability to scale-up, (semi)synthesis often suffers from producing fractions of unwanted stereo- or regio-specific isomers, particularly if blocking agents are not used, which can be costly and/or environmentally unfriendly. By contrast, enzymes can serve as regiospecific biocatalysts that maintain stereochemistry. With genetic engineering, enzymes can be expressed in heterologous hosts such as yeast or bacteria that have high expression potential, and can catalyze reactions using small molecule substrates fed through the culture medium. The shortfall of enzymes is that they are generally highly limited in the substrates that they can modify. However, some gene families commonly encode enzymes that are promiscuous (i.e. have broad substrate specificities) while being highly regiospecific. These include many plant UGTs (UDP:glycosedependent glycosyltransferases) or OMTs (O-methyltransferases). For example, UGT78K1 from soybean can transfer a hexose to the 3-0-position of at least 10 flavonol and anthocyanin molecules using UPD-glucose or UDP-galactose as a sugar donor [2]. GT83F from Medicago truncatula can transfer glucose to flavonoids and isoflavonoids when expressed in E. coli [3]. This raised the possibility that promiscuous enzymes could act on semi-synthetic substrates to make novel molecules for bioactivity testing.
We recently demonstrated that an OMT from rice, namely OsNOMT, can methylate the 7-0-position of a semi-synthetic dibrominated derivative of the NP apigenin, despite that its natural substrate is naringenin [4]. We further demonstrated that the efficiency to produce our target molecule 6,8-dibromogenkwanin could be changed depending on the order in which semi-synthesis and biocatalysis 'modules' were employed [4]. Based on this proof-of-concept, we propose that repositories of gene constructs that encode biocatalysts along with a database of their substrate specificities and semi-synthesis protocols could be assembled by the scientific community to facilitate the (bio)synthesis of 'recalcitrant' small molecules for bioactivity testing. We further propose that machine learning could be applied to the database to predict module order that should be used to provide highest yield of a target molecule. Scale-up for commercial production could then be achieved by directed evolution [5] of biocatalysis modules and optimized semi-synthesis methods.

\section{Acknowledgement}

This work was supported by WVU start-up funds to NK and was based upon work that is supported by the USDA National Institute of Food and Agriculture, Hatch, accession number 1010200, and the West Virginia Agricultural and Forestry Experiment Station project WVA00687.

\section{Conflicts of Interest}

No conflicts of interest. 


\section{References}

1. Newman DJ, Cragg GM (2016) Natural products as sources of new drugs from 1981 to 2014. J Nat Prod 79(3): 629-661.

2. Kovinich N, Saleem A, Arnason JT, Miki B (2010) Functional characterization of a UDP-glucose: flavonoid 3-0-glucosyltransferase from the seed coat of black soybean (Glycine max (L) Merr). Phytochemistry 71(11-12): 1253-1263.

3. Modolo LV, Blount JW, Achnine L, Naoumkina MA, Wang X, et al. (2007) A functional genomics approach to (iso) flavonoid glycosylation in the model legume Medicago truncatula. Plant molecular biology 64(5): 499518.

4. Gary S, Adegboye J, Popp B, Cocuron JC, Woodrum B, et al. (2018) Combining Semi-Synthesis with Plant and Microbial Biocatalysis: New Frontiers in Producing a Chemical Arsenal Against Cancer. RSC Advances 8(38): 21332-21339.

5. Cherry JR, Fidantsef AL (2003) Directed evolution of industrial enzymes an update. Current opinion in biotechnology 14(4): 438-443. 